\title{
Metformin inhibits the proliferation and metastasis of osteosarcoma cells by suppressing the phosphorylation of Akt
}

\author{
ZUOHONG LI ${ }^{1}$, LESHENG WANG $^{2}$, NAN LUO $^{3}$, YANTAO ZHAO $^{1}$, JIAZHI LI $^{4}$, QIWEI CHEN $^{4}$ and YU TIAN ${ }^{4}$ \\ ${ }^{1}$ Department of Orthopedics, Dalian Municipal Central Hospital, Dalian, Liaoning 116033; \\ ${ }^{2}$ Department of Blood Transfusion, Chinese People's Liberation Army 210 Hospital, Dalian, Liaoning 116015; \\ ${ }^{3}$ Department of Infectious Diseases, The Second Hospital of Dalian Medical University, Dalian, Liaoning 116027; \\ ${ }^{4}$ Department of Pathology, Dalian Medical University, Dalian, Liaoning 116044, P.R. China
}

Received June 6, 2016; Accepted August 17, 2017

DOI: $10.3892 / \mathrm{ol} .2018 .8297$

\begin{abstract}
Metformin (Met) is a therapeutic agent for the treatment of type 2 diabetes mellitus. There is evidence that Met may reduce the risk of cancer in patients with type 2 diabetes mellitus by inhibiting tumor cell growth, prolonging the overall survival time in patients with various types of malignancy. However, the function and mechanism of Met have not been fully elucidated in osteosarcoma (OS). The present study evaluated the anti-proliferative effect of Met on MG63 and U2OS OS cells, identifying that it acted in a dose- and time-dependent manner. Met also inhibited OS cell migration and invasion, potentially by regulating the epithelial-mesenchymal transition in OS cells. Mechanistically, Met was demonstrated to partly exert these functions through the suppression of Akt phosphorylation, which was associated with increased phosphatase and tensin (PTEN) expression. Silencing PTEN prevented the Met-induced inhibition of the growth and metastasis of OS cells. As Met has anti-proliferative and anti-metastatic effects on OS cells it is a potential candidate, in combination with other chemotherapeutic agents, for use in the treatment of OS.
\end{abstract}

\section{Introduction}

Osteosarcoma (OS) is the most common primary bone tumor in children and adolescents (1). OS is characterized by the local invasion of bone and soft tissues, with tissues exhibiting a relatively poor response to chemotherapy and radiotherapy, causing a high mortality rate $(2,3)$. Previous studies have

Correspondence to: Dr Zuohong Li, Department of Orthopedics, Dalian Municipal Central Hospital, 862 Xinan Road, Dalian, Liaoning 116033, P.R. China

E-mail: davelee2000@126.com

Dr Yu Tian, Department of Pathology, Dalian Medical University, 9 West Lvshunnan Road, Dalian, Liaoning 116044, P.R. China

E-mail: 64580413@qq.com

Key words: metformin, osteosarcoma, proliferation, metastasis, phosphatase and tensin homolog, Akt identified that $>20 \%$ of OS patients present with lung metastases at the point of initial diagnosis $(4,5)$. The 5 year survival rate is $\sim 65 \%$ for patients with localized OS; however, the rate for patients exhibiting metastatic disease is markedly lower, at $\sim 20 \%$ (6-8). Therefore, novel strategies for the treatment of OS are urgently required.

Metastasis is responsible for the majority of cancer-associated mortality and remains the most poorly understood component of cancer progression. Evidence indicates that the epithelial-mesenchymal transition (EMT) serves a vital role in the initiation of metastasis $(9,10)$. In this process, epithelial cells lose cell-cell adhesions and acquire the properties of mesenchymal cells, enhancing their migratory and invasive abilities (11). A range of growth factors are involved in the initiation of EMT, including the insulin-like growth factor-1 receptor (IGF-1R)/ligand system, which is reported to increase the metastatic potential of prostate and breast cancer cells $(12,13)$. Studies have consistently observed that the high local expression of IGF-1 in primary tumor tissue appears to substantially affect the aggressiveness of OS (14-16).

Phosphatidylinositol 3-kinase (PI3K) and Akt are aberrantly activated in OS (17). Clinical and preclinical studies have indicated that drugs targeting the PI3K/Akt pathway can be used to treat malignant tumors in the esophagus, lung, kidney and prostate, as well as those of the bone (18-22). A previous study has also indicated that inhibiting the PI3K/Akt pathway may inhibit OS invasion and migration (23).

Metformin (Met) is a biguanide antidiabetic agent widely used to treat type 2 diabetes mellitus (24). Studies have indicated that Met exhibits anti-proliferative properties and its use has been associated with a decreased incidence of cancer in diabetic patients (25-28). Met may also inhibit EMT in various other types of tumor $(29,30)$. However, its effect on OS is not yet well-characterized.

The present study aimed to identify the effect and mechanism of Met in OS progression. It was identified that Met inhibited the proliferation, migration and invasion of OS cells, which was accompanied with the upregulation of PTEN expression and the suppression of Akt expression. Silencing PTEN in OS cells prevented the Met-induced anti-growth and anti-metastatic effects. Therefore, Met may be useful as an adjuvant agent for OS therapy. 


\section{Materials and methods}

Cell culture and reagents. The human OS MG63 and U2OS cell lines were obtained from the American Type Culture Collection (Manassas, VA, USA). Cells were cultured in DMEM medium supplemented with $10 \%$ fetal bovine serum (FBS), $100 \mu \mathrm{g} / \mathrm{ml}$ penicillin and $100 \mu \mathrm{g} / \mathrm{ml}$ streptomycin (all Thermo Fisher Scientific, Inc., Waltham, MA, USA) in a 5\% $\mathrm{CO}_{2}$ atmosphere at $37^{\circ} \mathrm{C}$. Met (1,1-dimethylbiguanide hydrochloride) was purchased from Sigma-Aldrich (Merck KGaA, Darmstadt, Germany). The Cell Counting kit-8 (CCK-8) was purchased from Nanjing KeyGen Biotech Co., Ltd. (Nanjing, China). Antibodies against $\beta$-actin (cat. no. sc-47778), Akt (cat. no. sc-8312), phosphorylated (p)-Akt (cat. no. sc-33437) and phosphatase and tensin homolog (PTEN) (cat. no. sc-6817-R) were purchased from Santa Cruz Biotechnology, Inc. (Dallas, TX, USA), and anti-E-cadherin (cat. no. ab1416) from Abcam (Cambridge, UK); vimentin (cat. no. 10366-1-AP) was purchased from ProteinTech Group, Inc. (Chicago, IL, USA); the goat anti-rabbit (cat. no. 32460) and goat anti-mouse (cat. no. 32430) horseradish peroxidase (HRP)-conjugated secondary antibodies were purchased from Thermo Fisher Scientific, Inc.

RNA interference. PTEN small interfering (si)RNA and negative control (NC) siRNA were synthesized by Shanghai GenePharma Co., Ltd. (Shanghai, China). PTEN-siRNA, 5'-GATCTACTCCTCCAACTCA-3'; NC-siRNA, 5'-UUC UCCGAACGUGUCACGUTT-3'. At $24 \mathrm{~h}$ after seeding, the cells were transfected with PTEN-siRNA or NC-siRNA using Lipofectamine 2000 (Thermo Fisher Scientific, Inc.) according to the manufacturer's protocol. After $24 \mathrm{~h}$ transfection, cells were collected for the following experiments; $48 \mathrm{~h}$ after transfection, cells were collected for western blot analysis.

Cell viability assay. Cell viability was determined using the CCK-8 assay. MG63 and U2OS cells with or without PTENor NC-siRNA $\left(2 \times 10^{3}\right.$ per well $)$ were seeded into a 96 -well plate. At $24 \mathrm{~h}, 0,1,5$ or $10 \mathrm{mM}$ Met was added into the culture medium. Subsequent to incubation at $37^{\circ} \mathrm{C}$ for a further 24 or $48 \mathrm{~h}$, the medium was exchanged for $100 \mu \mathrm{l}$ DMEM medium and $10 \mu \mathrm{l}$ CCK- 8 reagent. The cells were incubated for $2 \mathrm{~h}$ at $37^{\circ} \mathrm{C}$. Finally, the optical density was measured using an EnSpire ${ }^{\mathrm{TM}} 2300$ Multilabel Reader (PerkinElmer, Inc., Waltham, MA, USA) at $450 \mathrm{~nm}$. Three replicates were prepared for each condition.

Colony formation assay. For the colony formation assay, MG63 and U2OS cells with or without PTEN- or NC-siRNA treated with $0,1,5$ or $10 \mathrm{mM}$ Met were seeded in triplicate at 1,000 cells/well in a 6 -well plate with complete medium. Subsequent to 3 weeks of growth, colonies were fixed with $4 \%$ paraformaldehyde for $10 \mathrm{~min}$ at room temperature and stained with $1 \%$ crystal violet for $15 \mathrm{~min}$ at room temperature $(0.1 \%$ $\mathrm{w} / \mathrm{v}$ in $20 \mathrm{nM}$ 4-morpholinepropanesulfonic acid). Visible colonies were counted.

Wound-healing assay. A total of $2 \times 10^{6} \mathrm{MG} 63$ and U2OS cells with or without PTEN- or NC-siRNA treated with $0,1,5$ or $10 \mathrm{mM}$ Met were seeded in 6-well plates, the cell monolayers were wounded by scratching with sterile plastic $20 \mu 1$ micropipette tips and images were captured using a light microscope at 0 and 24 or $48 \mathrm{~h}$. The migration distance of the cells was measured using Image Pro-Plus v.6.0 (Media Cybernetics, Inc., Rockville, MD, USA).

Invasion assay. The invasive capacity of MG63 and U2OS cells with or without PTEN- or NC-siRNA treated with 0 , 1,5 or $10 \mathrm{mM}$ Met was measured with Matrigel-coated (BD Biosciences, Franklin Lakes, NJ, USA) Transwell inserts (Costar, Manassas, VA, USA). The inserts were coated with $50 \mu \mathrm{l}$ of $1 \mathrm{mg} / \mathrm{ml}$ Matrigel according to the manufacturer's protocol. A total of $2 \times 10^{5}$ cells in $200 \mu \mathrm{l}$ DMEM serum-free medium were plated in the upper chamber, with $300 \mu \mathrm{l}$ of medium with $10 \%$ FBS added to the lower chamber. Following a $24 \mathrm{~h}$ incubation, cells that invaded to the lower surface of the membrane were fixed and stained. For each membrane, five random fields of view were counted.

Western blotting analysis. MG63 and U2OS cells with or without PTEN- or NC-siRNA treated with $0,1,5$ or $10 \mathrm{mM}$ Met were harvested and lysed in radioimmunoprecipitation assay buffer (Nanjing KeyGen Biotech Co., Ltd.) supplemented with $1 \mathrm{mM}$ phenylmethylsulfonyl fluoride and $1 \mathrm{mM}$ phosphatase inhibitor cocktail. The protein concentration was determined using a bicinchoninic assay kit (Nanjing KeyGen Biotech Co., Ltd.). A total of $30 \mu \mathrm{g}$ protein samples were mixed with loading buffer, and the proteins were separated using $10 \%$ SDS-PAGE and transferred to polyvinylidene difluoride membranes (EMD Millipore, Billerica, MA, USA). Subsequent to blocking in 5\% nonfat dry milk for $2 \mathrm{~h}$ at room temperature, the blots were incubated at $4^{\circ} \mathrm{C}$ overnight with the aforementioned primary antibodies $(1: 1,000)$ and at $37^{\circ} \mathrm{C}$ for $2 \mathrm{~h}$ with the secondary antibodies $(1: 20,000)$. The bands were visualized with WesternBright Sirius HRP substrate (Advansta, Inc., Menlo Park, CA, USA) and were imaged using a Vilber Fusion FX5 (Vilber Lourmat, Marne-la-Valée, France).

Statistical analysis. SPSS 16.0 software (SPSS, Inc., Chicago, IL, USA) was used to perform statistical analysis. Values are presented as the mean \pm standard deviation. The statistical analysis of differences between two groups was performed using Student's t-test and the analysis of multiple groups was performed using one-way analysis of variance followed by the Student-Newman-Keuls post hoc test. $\mathrm{P}<0.05$ was considered to indicate a statistically significant difference.

\section{Results}

Met inhibits the proliferation of OS cells. To investigate the effect of Met on OS cell proliferation, CCK- 8 analysis was employed to assess the viability of MG63 and U2OS cells. As presented in Fig. 1A and B, the groups treated with 1,5 and $10 \mathrm{mM}$ Met exhibited a dose- and time-dependent inhibition of proliferation. Consistent with the results from the CCK-8 assay, the colony formation rates in the cells treated with Met were lower than the control group, particularly in the 5 and $10 \mathrm{mM}$ Met groups $(\mathrm{P}<0.01$; Fig. $1 \mathrm{C}$ and $\mathrm{D})$. These data indicated that Met inhibited the growth of OS cells. 
A
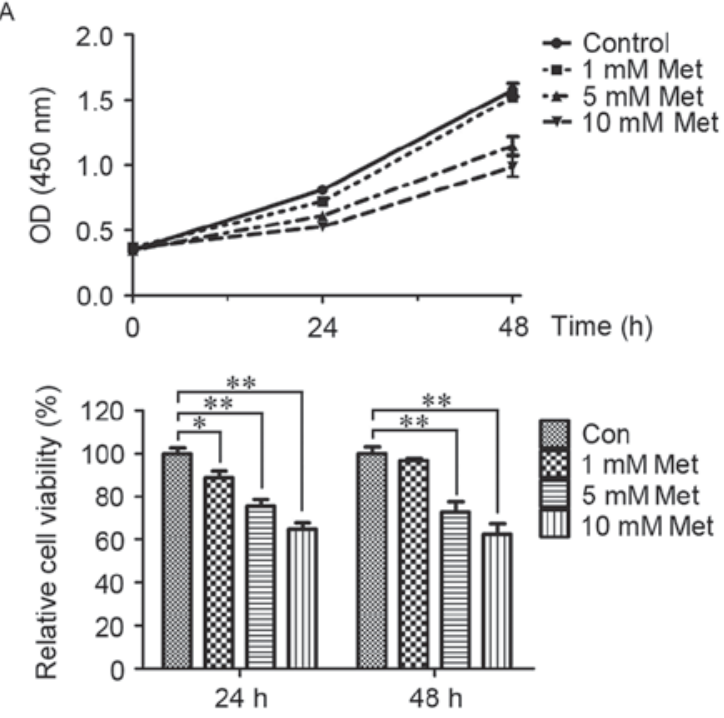

C
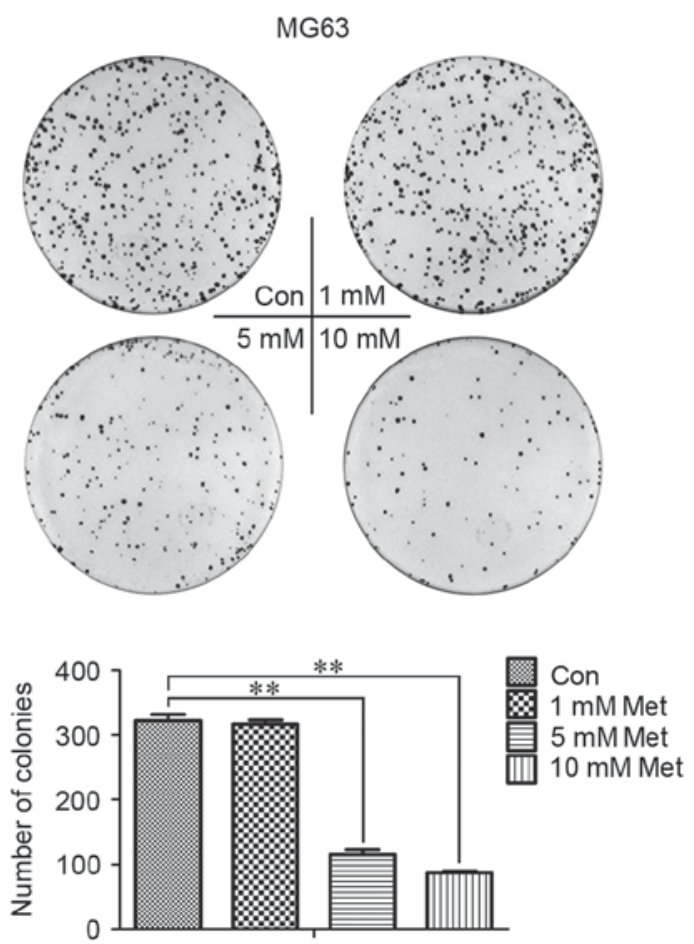

$B$
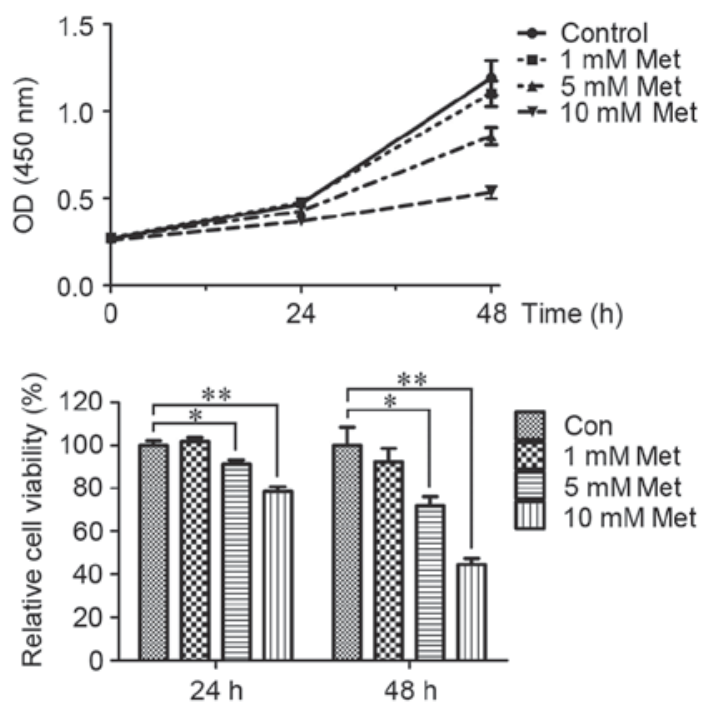

D
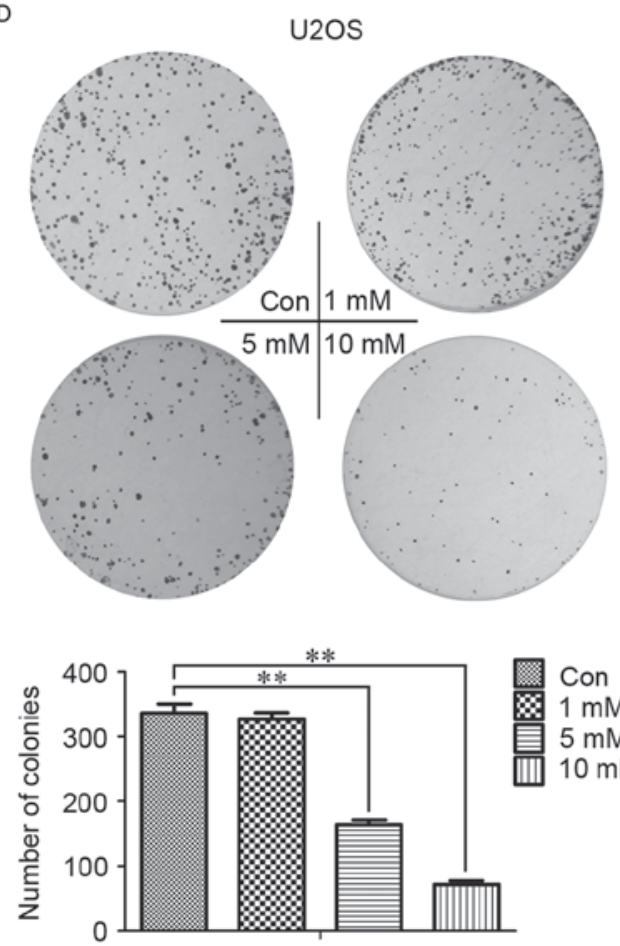

Con

$1 \mathrm{mM}$ Met

$5 \mathrm{mMMet}$

$10 \mathrm{mM}$ Met

Figure 1. Met inhibits the proliferation of OS cells. Cell Counting kit-8 analysis was utilized to evaluate the proliferation of (A) MG63 and (B) U2OS OS cells treated with the indicated concentrations of Met for 0,24 and 48 h. (C) MG63 and (D) U2OS cells were plated in 6-well plates at a density of 1,000 cells/well with the indicated concentrations of Met. At 2 weeks, the number of colonies was counted. ${ }^{*} \mathrm{P}<0.05,{ }^{*} \mathrm{P}<0.01$. Met, metformin; OS, osteosarcoma; Con, control (0 mM Met); OD, optical density.

Met suppresses the migration and invasion ability of OS cells. To examine the effect of Met on the mobility of MG63 cells, wound-healing and invasion assays were utilized. The speed of wound closure was reduced with increasing concentrations of Met, compared with the control group (Fig. 2A and B). Incubation with Met also suppressed the invasion of MG63 and $\mathrm{U} 2 \mathrm{OS}$ cells in a dose-dependent manner (Fig. 2C and D). As EMT is a critical mechanism in the progression of tumor cells to metastasis, whether Met could negatively regulate the EMT of OS cells was analyzed. Following treatment with $10 \mathrm{mM}$ Met, MG63 and U2OS cells exhibited increased levels of the epithelial marker E-cadherin and decreased levels of the mesenchymal marker vimentin (Fig. 2E), which indicated that Met may suppress the metastasis of OS cells by negatively regulating EMT.

Met inhibits the proliferation, migration and invasion of OS cells by suppressing the phosphorylation of Akt. The molecular mechanisms responsible for the proliferation, migration and invasion suppressive effect of Met were assessed. Akt serves a key role in regulating the biological functions of tumor cells, including proliferation, apoptosis and metastasis; PTEN is a regulator that inhibits the activation of Akt (31). Following treatment with $10 \mathrm{mM}$ Met for $48 \mathrm{~h}$, the expression level of PTEN, Akt and p-Akt were detected by western blotting. As demonstrated in Fig. 3A, the expression level of PTEN was upregulated 
A
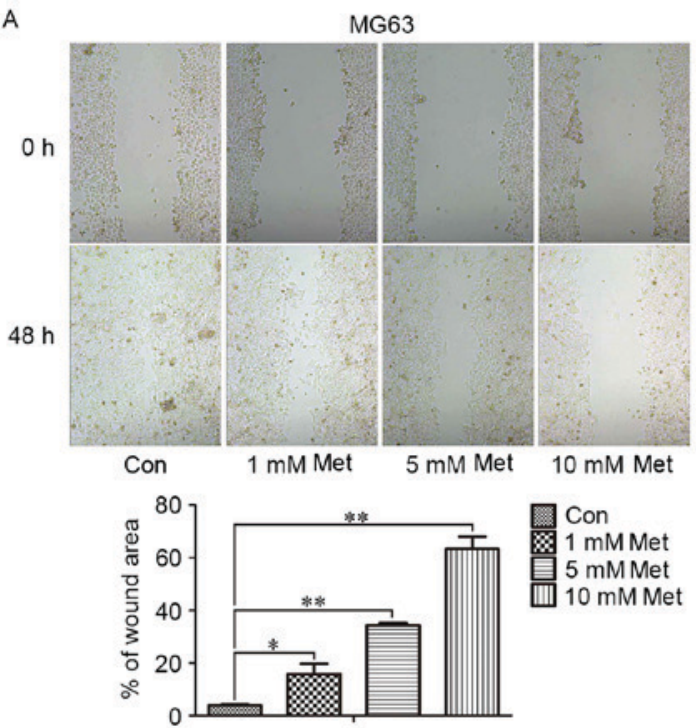

C
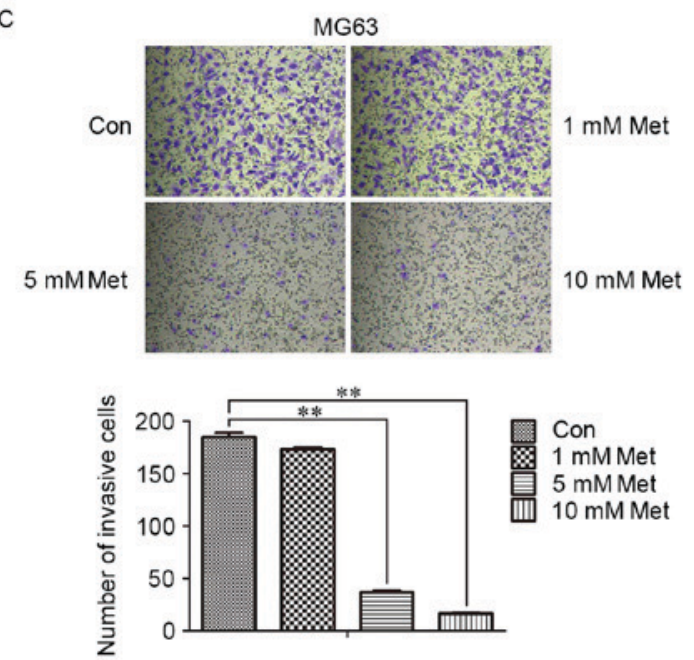

E

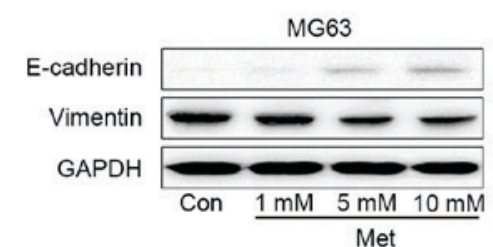

B
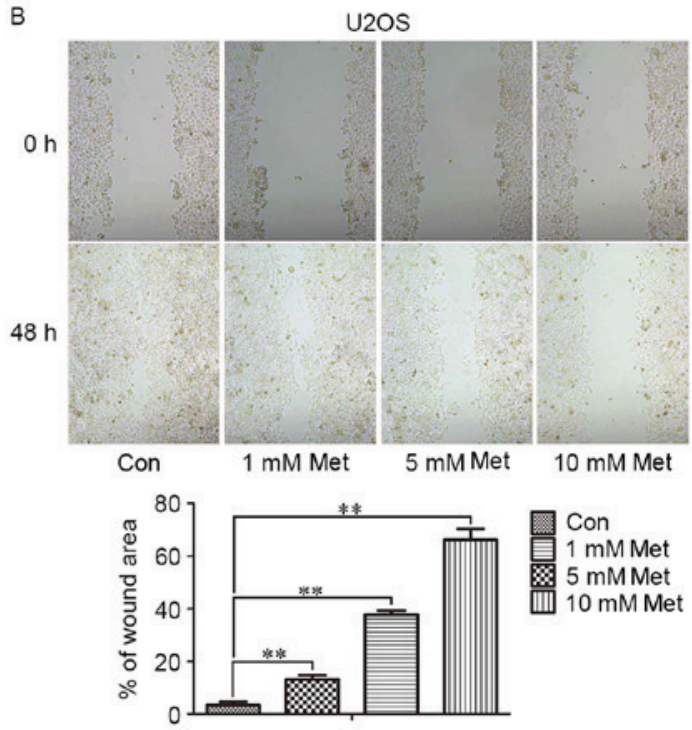

D
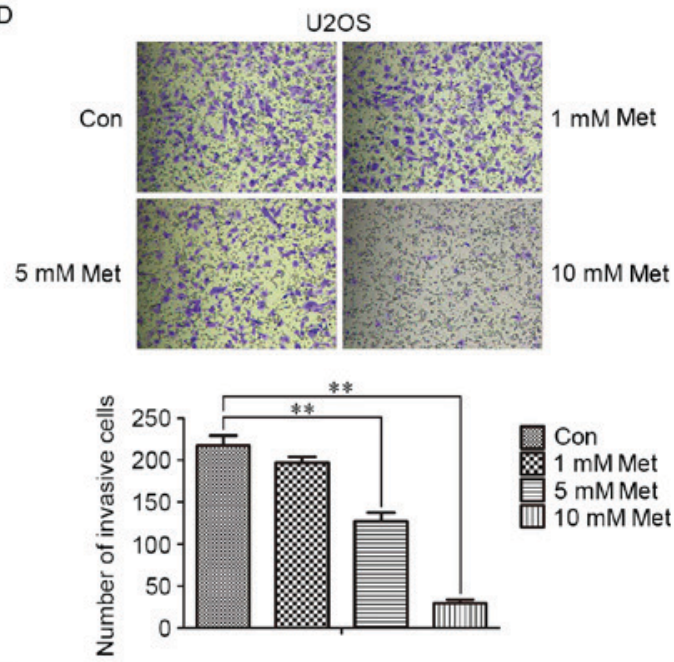

F

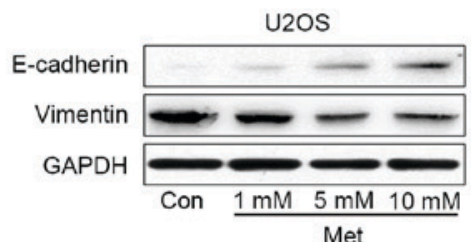

Figure 2. Met inhibits the migration and invasion of osteosarcoma cells. The wound area was measured prior to and at $48 \mathrm{~h}$ after the treatment of (A) MG63 and (B) U2OS cells with the indicated concentrations of Met (magnification, x400). The number of invading (C) MG63 and (D) U2OS cells in Matrigel-coated Transwell inserts were counted at $24 \mathrm{~h}$ after plating (magnification, x400). Representative images of western blotting results for epithelial-mesenchymal transition-associated markers, including E-cadherin and vimentin, in (E) MG63 and (F) U2OS cells following the indicated treatments are presented. "P<0.05, ${ }^{* * *} \mathrm{P}<0.01$. Met, metformin; Con, control (0 mM Met).
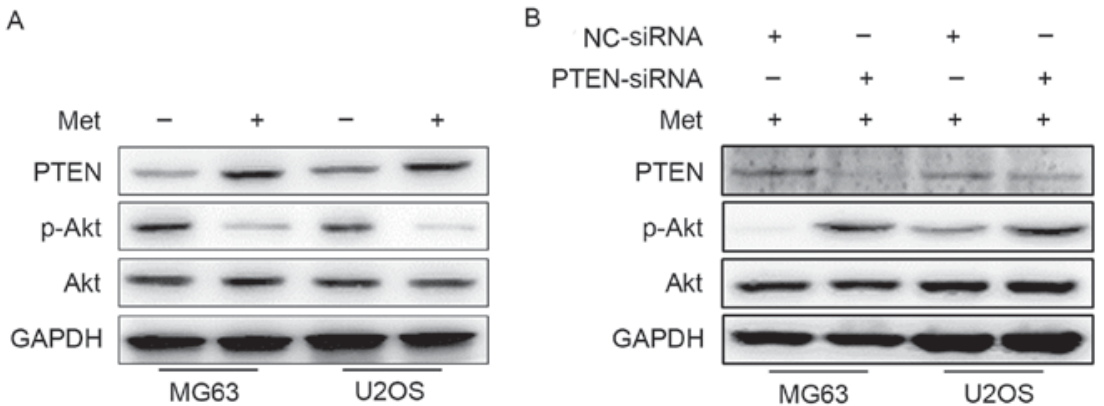

Figure 3. Met suppresses the activation of Akt through the upregulation of PTEN. (A) Representative images of western blotting results for PTEN, p-Akt and Akt in MG63 and U2OS cells with or without $48 \mathrm{~h}$ of $10 \mathrm{mM}$ Met treatment. (B) With the same treatment, the protein levels of PTEN, p-Akt and Akt were measured in NC-siRNA- and PTEN-siRNA-transfected osteosarcoma cells. Met, metformin; PTEN, phosphatase and tensin homolog; p-, phosphorylated; NC, negative control; siRNA, small interfering RNA. 
A

MG63

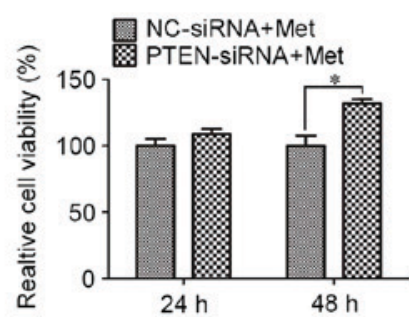

c
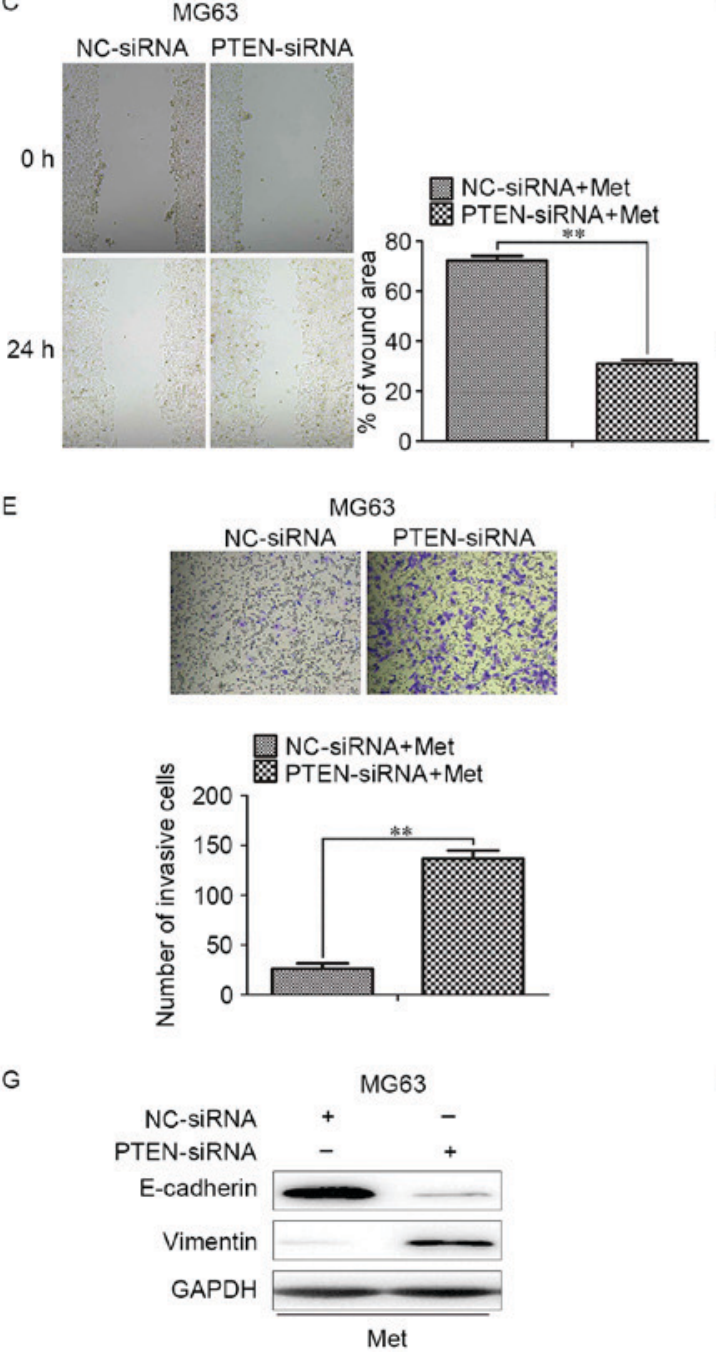

B

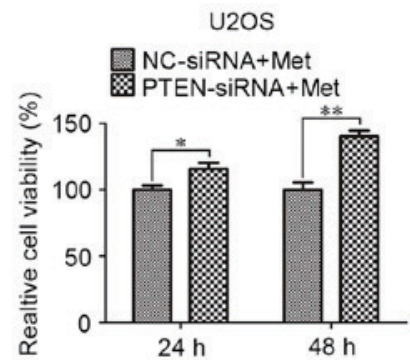

D

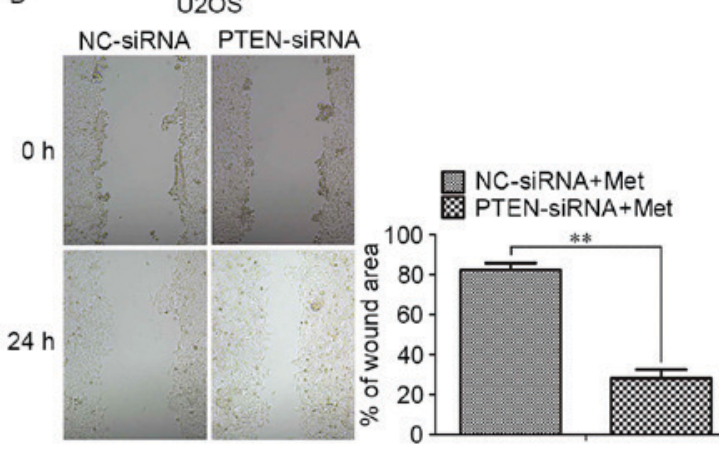

$\mathrm{F}$

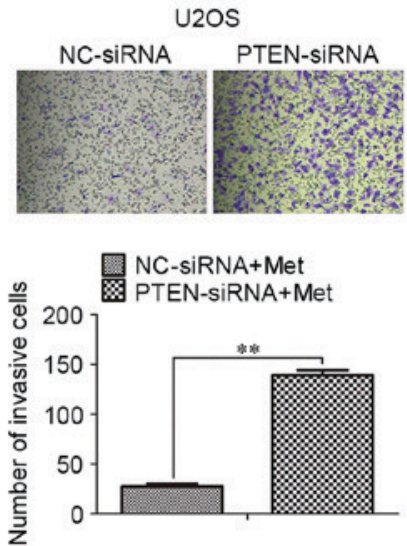

H

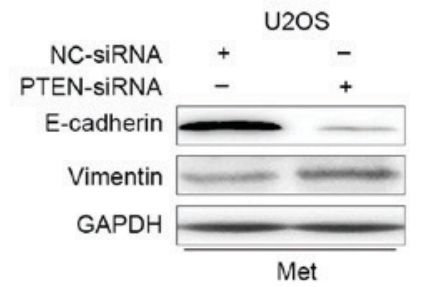

Figure 4. Silencing PTEN blocks Met-induced anti-growth and anti-metastasis effects on OS cells. OS cells transfected with PTEN- or NC-siRNA were treated with $10 \mathrm{mM}$ Met for $48 \mathrm{~h}$. Relative cell viability of (A) MG63 and (B) U2OS cells with the indicated treatments, Wound healing assays for (C) MG63 and (D) U2OS cells with the indicated treatments (magnification, $\mathrm{x} 400$ ). Transwell assays for (E) MG63 and (F) U2OS cells with the indicated treatments (magnification, $\mathrm{x} 400$ ). Representative images of western blots for epithelial-mesenchymal transition-associated markers, including E-cadherin and vimentin, in (G) MG63 and (H) U2OS cells. ${ }^{~} \mathrm{P}<0.05,{ }^{* *} \mathrm{P}<0.01$. PTEN, phosphatase and tensin homolog; Met, metformin; OS, osteosarcoma; NC, negative control; siRNA, small interfering RNA.

following this treatment, whereas the phosphorylation of Akt was downregulated. These data indicated that Met may inhibit the proliferation, migration and invasion of OS cells by suppressing the phosphorylation of Akt through the upregulation of PTEN.

To provide further confirmation that Met exerts its function through the PTEN/Akt pathway, MG63 and U2OS cells were transfected with NC- or PTEN-siRNA prior to Met treatment, and western blotting was performed. The expression of PTEN was confirmed to be lower, and the phosphorylation of Akt, higher, in cells transfected with PTEN-siRNA compared with NC-siRNA-transfected cells (Fig. 3B).
Next, the proliferative, migratory and invasive capacities of cells transfected with PTEN-siRNA in response to Met treatment were assessed. With Met treatment, MG63 and U2OS cells transfected with PTEN-siRNA exhibited an increased rate of proliferation when compared with the corresponding NC-siRNA cells (Fig. 4A and B). The inhibition of migration and invasion in MG63 and U2OS cells were also reversed by PTEN-knockdown (Fig. 4C and D). These data suggest that PTEN/Akt is important for the Met-mediated suppression of proliferation, invasion and metastasis in OS cells. 


\section{Discussion}

OS remains a devastating disease, and its treatment is a major challenge in oncology (32). Chemotherapeutic treatments are often the first choice for OS therapy despite the high cost of the drugs and the high incidence of drug resistance and side effects (33). Met is a therapeutic agent used to treat type 2 diabetes mellitus. Met has been reported as an effective adjuvant drug in the treatment of OS (34). However, the mechanism by which Met inhibits OS growth and metastasis have yet to be established. The present study demonstrated that Met inhibited the growth and metastasis of OS cells by suppressing the phosphorylation of Akt by upregulating the expression of PTEN.

To evaluate the effect of Met on OS cells, the viability of MG63 and U2OS cells treated with 1, 5 and $10 \mathrm{mM} \mathrm{Met}$ was determined in comparison to a control group. The cell viability was inhibited more significantly in the $10 \mathrm{mM} \mathrm{Met}$ group than in the other treatment groups, and the inhibitory effects increased in a dose- and time-dependent manner. Additionally, the colony formation capability was decreased upon treatment with Met.

As OS frequently metastasizes, strategies for inhibiting metastasis may be critical for treating this malignancy. Met has been reported to suppress the metastasis-associated behaviors of migration and invasion in certain types of malignancy $(29,30)$; however, to the best of our knowledge, whether Met affects these behaviors in OS has yet to be determined. Therefore, the present study evaluated the anti-metastatic effects of Met in MG63 and U2OS cells using wound-healing and invasion assays with a range of doses of Met. Similar to the previous studies, the ability of OS cells to migrate and invade was inhibited, particularly following treatment with $10 \mathrm{mM}$ Met.

EMT is characterized by the decreased expression of epithelial markers, the increased expression of mesenchymal markers, alterations to the morphology of cells, loss of cellular adhesion and advancement in cell motility. EMT contributes to the invasion and metastasis of cancer cells, and is a key event in the progression of numerous types of cancer. Multiple transcription factors, including Snail, Twist and Zeb, regulate the expression of EMT-associated markers. A variety of molecules can induce EMT, including transforming growth factor- $\beta$, epidermal growth factor, Akt and extracellular-regulated kinases $(35,36)$. However, to the best of our knowledge, the Met-mediated alteration to EMT marker expression in OS cells has not been previously reported. In the present study, treatment with Met promoted the transition of MG63 and U2OS cells from a mesenchymal to an epithelial phenotype, as characterized by the increased expression of an epithelial marker and decreased expression of a mesenchymal marker. These findings confirmed that Met treatment may inhibit metastasis by regulating the EMT of OS cells.

Aberrant activation of various signaling pathways, including the PTEN/Akt pathway, appears to have a major role in the pathogenesis of OS. Phosphorylation of Akt at Ser473 can activate downstream effectors, which can activate GSK-3 $\beta$ and lead to the reduced phosphorylation and ubiquitination of Snail (37), causing the accumulation of Snail in the nucleus, which transactivates the expression of mesenchymal markers and inhibits the transcription of E-cadherin (38). PTEN, a major negative regulator of the PI3K/Akt pathway, can inhibit the phosphorylation of Akt by targeting its 3'-untranslated region. Loss of PTEN activity is frequently observed in OS (39). Therefore, this complex pathway is considered to be one of the most attractive targets for OS treatment; however, few therapies exploiting this have entered clinical trials (40). In the present study, Met was demonstrated to act as an anti-OS agent via the upregulation of PTEN and downregulation of Akt phosphorylation, with the knockdown of PTEN preventing the Met-mediated inhibition of OS cell growth and metastatic behaviors.

In conclusion, the present study has identified mechanisms by which Met, an inexpensive and widely used antidiabetic drug without severe adverse effects, may inhibit the proliferation, migration and invasion of OS cells; Met may suppress the phosphorylation of Akt through the upregulation of PTEN expression. These findings indicate that Met may be a potential candidate for clinical therapy, potentially applied in combination with other chemotherapeutic agents, to treat OS. However, further investigation may be necessary to evaluate its applicability in the overall strategy for OS therapy.

\section{Acknowledgements}

Not applicable.

\section{Funding}

No funding was received.

\section{Availability of data and materials}

Data used during the current study are available from the corresponding author on reasonable request.

\section{Authors' contributions}

YT and ZL designed the study. YT, ZL, LW, NL, YZ, JL and QC performed the experiments and data analysis. YT produced the figures, and wrote the initial draft manuscript draft. ZL edited the final draft manuscript.

\section{Ethics approval and consent to participate}

Not applicable.

\section{Consent for publication}

Not applicable.

\section{Competing interests}

The authors declare that they have no competing interests.

\section{References}

1. Moore DD and Luu HH: Osteosarcoma. Cancer Treat Res 162: 65-92, 2014.

2. Chou AJ and Gorlick R: Chemotherapy resistance in osteosarcoma: Current challenges and future directions. Expert Rev Anticancer Ther 6: 1075-1085, 2006.

3. Ma J, Niu M, Yang W, Zang L and Xi Y: Role of relaxin-2 in human primary osteosarcoma. Cancer Cell Int 13: 59, 2013. 
4. Bacci G, Rocca M, Salone M, Balladelli A, Ferrari S, Palmerini E, Forni C and Briccoli A: High grade osteosarcoma of the extremities with lung metastases at presentation: Treatment with neoadjuvant chemotherapy and simultaneous resection of primary and metastatic lesions. J Surg Oncol 98: 415-420, 2008.

5. Messerschmitt PJ, Garcia RM, Abdul-Karim FW, Greenfield EM and Getty PJ: Osteosarcoma. J Am Acad Orthop Surg 17: 515-527, 2009.

6. Bielack SS, Kempf-Bielack B, Delling G, Exner GU, Flege S, Helmke K, Kotz R, Salzer-Kuntschik M, Werner M, Winkelmann W, et al: Prognostic factors in high-grade osteosarcoma of the extremities or trunk: An analysis of 1,702 patients treated on neoadjuvant cooperative osteosarcoma study group protocols. J Clin Oncol 20: 776-790, 2002.

7. Eccles SA and Welch DR: Metastasis: Recent discoveries and novel treatment strategies. Lancet 369: 1742-1757, 2007.

8. Hughes DP: Strategies for the targeted delivery of therapeutics for osteosarcoma. Expert Opin Drug Deliv 6: 1311-1321, 2009.

9. Kerosuo L and Bronner-Fraser M: What is bad in cancer is good in the embryo: Importance of EMT in neural crest development. Semin Cell Dev Biol 23: 320-332, 2012.

10. Khan MA, Chen HC, Zhang D and Fu J: Twist: A molecular target in cancer therapeutics. Tumour Biol 34: 2497-2506, 2013.

11. Thompson EW, Newgreen DF and Tarin D: Carcinoma invasion and metastasis: A role for epithelial-mesenchymal transition? Cancer Res 65: 5991-5995, 2005.

12. Walsh LA and Damjanovski S: IGF-1 increases invasive potential of MCF 7 breast cancer cells and induces activation of latent TGF- $\beta 1$ resulting in epithelial to mesenchymal transition. Cell Commun Signal 9: 10, 2011.

13. Graham TR, Zhau HE, Odero-Marah VA, Osunkoya AO, Kimbro KS, Tighiouart M, Liu T, Simons JW and O'Regan RM: Insulin-like growth factor-I-dependent up-regulation of ZEB1 drives epithelial-to-mesenchymal transition in human prostate cancer cells. Cancer Res 68: 2479-2488, 2008.

14. Jentzsch T, Robl B, Husmann M, Bode-Lesniewska B and Fuchs B: Worse prognosis of osteosarcoma patients expressing IGF-1 on a tissue microarray. Anticancer Res 34: 3881-3889, 2014

15. Jentzsch T, Robl B, Husmann M, Bode-Lesniewska B and Fuchs B: Worse prognosis of osteosarcoma patients expressing IGF-1 on a tissue microarray. Anticancer Res 34: 3881-3889, 2014.

16. de Groot S, Gelderblom H, Fiocco M, Bovée JV, van der Hoeven JJ, Pijl H and Kroep JR: Serum levels of IGF-1 and IGF-BP3 are associated with event-free survival in adult Ewing sarcoma patients treated with chemotherapy. Onco Targets Ther 10: 2963-2970, 2017.

17. Zhang J, Yu XH, Yan YG, Wang $\mathrm{C}$ and Wang WJ: PI3K/Akt signaling in osteosarcoma. Clin Chim Acta 444: 182-192, 2015.

18. Chen J, Lan T, Zhang W, Dong L, Kang N, Fu M, Liu B, Liu K, Zhang C, Hou J and Zhan Q: Dasatinib enhances cisplatin sensitivity in human esophageal squamous cell carcinoma (ESCC) cells via suppression of PI3K/AKT and Stat3 pathways. Arch Biochem Biophys 575: 38-45, 2015.

19. Wu H, Fan F, Liu Z, Shen C, Wang A and Lu Y: Norcantharidin combined with EGFR-TKIs overcomes HGF-induced resistance to EGFR-TKIs in EGFR mutant lung cancer cells via inhibition of Met/PI3k/Akt pathway. Cancer Chemother Pharmacol 76: 307-315, 2015

20. Zhu Y, Xu L, Zhang J, Xu W, Liu Y, Yin H, Lv T, An H, Liu L, $\mathrm{He} \mathrm{H}$, et al: Klotho suppresses tumor progression via inhibiting PI3K/Akt/GSK3 $3 /$ Snail signaling in renal cell carcinoma. Cancer Sci 104: 663-671, 2013

21. Yamamoto Y, De Velasco MA, Kura Y, Nozawa M, Hatanaka Y, Oki T, Ozeki T, Shimizu N, Minami T, Yoshimura K, et al: Evaluation of in vivo responses of sorafenib therapy in a preclinical mouse model of PTEN-deficient of prostate cancer. J Trans Med 13: 150, 2015

22. Liang C, Li H, Shen C, Lai J, Shi Z, Liu B and Tao HM: Genistein potentiates the anti-cancer effects of gemcitabine in human osteosarcoma via the downregulation of Akt and nuclear factor- $\kappa \mathrm{B}$ pathway. Anticancer Agents Med Chem 12: 554-563, 2012.
23. Zhu LB, Jiang J, Zhu XP, Wang TF, Chen XY, Luo QF, Shu Y, Liu ZL and Huang SH: Knockdown of Aurora-B inhibits osteosarcoma cell invasion and migration via modulating PI3K/Akt/ NF- $\mathrm{B}$ signaling pathway. Int J Clin Exp Pathol 7: 3984-3991, 2014

24. Olt S and Oznas O: Investigation of the vitamin B12 deficiency with peripheral neuropathy in patients with type 2 diabetes mellitus treated using metformin. North Clin Istanb 4: 233-236, 2017.

25. Cho SW, Yi KH, Han SK, Sun HJ, Kim YA, Oh BC, Park YJ and Park DJ: Therapeutic potential of metformin in papillary thyroid cancer in vitro and in vivo. Mol Cell Endocrinol 393: 24-29, 2014.

26. Lengyel E, Litchfield LM, Mitra AK, Nieman KM, Mukherjee A, Zhang Y, Johnson A, Bradaric M, Lee W and Romero IL: Metformin inhibits ovarian cancer growth and increases sensitivity to paclitaxel in mouse models. Am J Obstet Gynecol 212: 479.e471-479.e410, 2015.

27. Lau YK, Du X, Rayannavar V, Hopkins B, Shaw J, Bessler E, Thomas T, Pires MM, Keniry M, Parsons RE, et al: Metformin and erlotinib synergize to inhibit basal breast cancer Oncotarget 5: 10503-10517, 2014.

28. Ben Sahra I, Le Marchand-Brustel Y, Tanti JF and Bost F: Metformin in cancer therapy: A new perspective for an old antidiabetic drug? Mol Cancer Ther 9: 1092-1099, 2010.

29. Zhang J, Shen C, Wang L, Ma Q, Xia P, Qi M, Yang M and Han B: Metformin inhibits epithelial-mesenchymal transition in prostate cancer cells: Involvement of the tumor suppressor miR30a and its target gene SOX4. Biochem Biophys Res Commun 452: 746-752, 2014.

30. Zhao Z, Cheng X, Wang Y, Han R, Li L, Xiang T, He L, Long H, Zhu B and He Y: Metformin inhibits the IL-6-induced epithelial-mesenchymal transition and lung adenocarcinoma growth and metastasis. PloS One 9: e95884, 2014.

31. Gao F, Huang W, Zhang Y, Tang S, Zheng L, Ma F, Wang Y, Tang $\mathrm{H}$ and $\mathrm{Li} \mathrm{X}$ : Hes1 promotes cell proliferation and migration by activating Bmi-1 and PTEN/Akt/GSK3 $\beta$ pathway in human colon cancer. Oncotarget 6: 38667-38680, 2015.

32. Yang G, Yuan J and Li K: EMT transcription factors: Implication in osteosarcoma. Med Oncol 30: 697, 2013.

33. Lindsey BA, Markel JE and Kleinerman ES: Osteosarcoma overview. Rheumatol Ther 4: 25-43, 2017.

34. Duo J, Ma Y, Wang G, Han X and Zhang C: Metformin synergistically enhances antitumor activity of histone deacetylase inhibitor trichostatin a against osteosarcoma cell line. DNA Cell Biol 32: 156-164, 2013.

35. Mimeault M and Batra SK: Interplay of distinct growth factors during epithelial mesenchymal transition of cancer progenitor cells and molecular targeting as novel cancer therapies. Ann Oncol 18: 1605-1619, 2007.

36. Xu X, Fan Z, Kang L, Han J, Jiang C, Zheng X, Zhu Z, Jiao H, Lin J, Jiang K, et al: Hepatitis B virus X protein represses miRNA-148a to enhance tumorigenesis. J Clin Invest 123: 630-645, 2013

37. Zhou BP, Deng J, Xia W, Xu J, Li YM, Gunduz M and Hung MC: Dual regulation of Snail by GSK-3beta-mediated phosphorylation in control of epithelial-mesenchymal transition. Nat Cell Biol 6: 931-940, 2004

38. Cano A, Pérez-Moreno MA, Rodrigo I, Locascio A, Blanco MJ, del Barrio MG, Portillo F and Nieto MA: The transcription factor snail controls epithelial-mesenchymal transitions by repressing E-cadherin expression. Nat Cell Biol 2: 76-83, 2000.

39. Nielsen-Preiss SM, Silva SR and Gillette JM: Role of PTEN and Akt in the regulation of growth and apoptosis in human osteoblastic cells. J Cell Biochem 90: 964-975, 2003.

40. Li T, Xiao Y and Huang T: HIF1 $\alpha$ induced upregulation of lncRNA UCA1 promotes cell growth in osteosarcoma by inactivating the PTEN/AKT signaling pathway. Oncol Rep 39: 1072-1080, 2018 